\title{
The Relationship Between Self-Esteem and Loneliness: Does Social Anxiety Matter?
}

\author{
Ze-wei Ma ${ }^{1,2}$, Jia-jie Liang ${ }^{2}$, Wei-nan Zeng ${ }^{2}$, Su Jiang $^{1} \&$ Tian Liu ${ }^{2}$ \\ ${ }^{1}$ Thye Hua Social Work Service Center, Luogang District, Guangzhou, China \\ ${ }^{2}$ Department of Psychology, School of Humanities and Management, Guangdong Medical College, Dongguan, \\ China \\ Correspondence: Wei-nan Zeng, Department of Psychology, School of Humanities and Management, Guangdong \\ Medical College, Dongguan 523808, China. Tel: 86-139-2580-7930. E-mail: weinanzeng@hotmail.com
}

Received: April 17, 2014

Accepted: May 20, $2014 \quad$ Online Published: May 26, 2014

doi:10.5539/ijps.v6n2p151

URL: http://dx.doi.org/10.5539/ijps.v6n2p151

\begin{abstract}
An inverse relationship between self-esteem and loneliness has been established in previous research. In the current study, the hypothesis that social anxiety plays a mediated role between self-esteem and loneliness was investigated. A total of 285 Chinese college students were enrolled and measured on a series of questionnaires to measure self-esteem, social anxiety and loneliness. The causal steps approach indicated that social anxiety partially mediated the relationship between self-esteem and loneliness. Furthermore, the Bootstrapping 95\% confidence intervals as well as the Bayesian 95\% credible intervals indicated that the indirect effect was significant. In addition, both the kappa-squared and R-squared mediation effect size measures indicated that the effect size of the mediating effect was medium, and this result was confirmed by the other four effect size measures, the partially standardized indirect effect, the completely standardized indirect effect, the proportion mediated and the ratio effect size measure.
\end{abstract}

Keywords: loneliness, bootstrapping, Markov chain Monte Carlo, effect size measure

\section{Introduction}

Four decades ago, a seminal work about loneliness by Weiss was published, and a substantial increase in research on loneliness has been evoked (Russell, 1996). Obviously, every one of us has the experience of feeling lonely at certain points in our lives (Asher \& Paquette, 2003; Rokach \& Brock, 1997). The prevalence rate of loneliness in the general population is about $20 \%$ to $28 \%$ (Cacioppo \& Patrick, 2008; Oshagan \& Allen, 1992). From being an adolescent to being an adult and as a transitional period from high school to college, it is no wonder that college students are haunted by loneliness (Cutrona, 1982; O'Donovan \& Hughes, 2007; Vanhalst, 2012). Research from McWhirter showed that about $30 \%$ of college students experienced loneliness and at least $6 \%$ considered it a major problem (McWhirter, 1997). In Knox's study, 25.9\% of college male students and $16.7 \%$ of female college students were found to have severe lonely feelings (Knox, Vail-Smith, \& Zusman, 2007).

As a basic and universal emotion and subjective psychological experience, loneliness is always very painful, and it is composed of trouble and distress (Jones, Freemon, \& Goswick, 1981; Peplau \& Cutrona, 1980; Rokach, 2011). Loneliness is found to be a significant predictor of numerous clinically relevant problems (Heinrich \& Gullone, 2006), including poorer physical health and higher health care usage (Masi, Chen, Hawkley, \& Cacioppo, 2011) as well as insufficient social skills and sociability (Coplan, Closson, \& Arbeau, 2007). Although researchers have been trying to answer why we feel lonely, there still no agreement on this issue. However, empirical findings consistently indicated that the low self-esteem, which has been proven as one of the prominent cognitive characteristics of those lonely individuals, is associated with loneliness and low self-esteem is also thought to be an antecedent cause of loneliness (Brage, Meredith, \& Woodward, 1993; Hymel, Rubin, Rowden, \& LeMare, 1990; LARSON, 1999; Olmstead, Guy, O’Mally, \& Bentler, 1991; Peplau, Perlman, \& Perlman, 1982; Schultz \& Moore, 1988). It is likely that children who develop low self-esteem suffer more loneliness (Fordham \& Stevenson-Hinde, 1999; Kirova-Petrova, 2000). Additionally, numerous empirical studies with adolescents and adults have also linked poor self-esteem to loneliness (Creemers, Scholte, Engels, Prinstein, \& Wiers, 2012; Cutrona, 1982; Davis, Hanson, Edson, \& Ziegler, 1992; Kong \& You, 2013; 
McWhirter, 1997; McWhirter, Besett-Alesch, Horibata, \& Gat, 2002; Nurmi, Toivonen, Salmela-Aro, \& Eronen, 1997).

Even though the inverse relationship between self-esteem and loneliness has been well investigated, knowledge on the explanatory or potential mechanisms linking self-esteem to loneliness remains limited. One central goal of science is to understand how processes work rather than simply to establish whether a total effect exists (Hayes \& Preacher, 2010). In other words, it is necessary to investigate whether the inverse relationship between self-esteem and loneliness is accounted for by any intervening variables.

It has been well established that how feelings about one's self affect one's behavior and social interactions (Mecca, Smelser, \& Vasconcellos, 1989). Individuals with high self-esteem enjoy communal interactions while those with low self-esteem have low confidence and do not feel comfortable in social interactions (Sarason, Sarason, \& Pierce, 1990). Furthermore, empirical findings suggest that the low self-esteem is a risk factor for the social anxiety (Clark \& Beck, 2011; De Jong, Sportel, De Hullu, \& Nauta, 2012). The first identifiable study investigating the relationship between self-esteem and social anxiety was conducted by Geist and Borecki $($ Chrystan, 2007) $=$ This study showed that people with high social anxiety have lower levels of self-esteem. According to Cox, people with social anxiety are typified by low self-esteem and high self-criticism (Cox, Fleet, \& Stein, 2004). Studies have confirmed that self-esteem and social anxiety shares a significant inverse relationship (Obeid, Buchholz, Boerner, Henderson, \& Norris, 2013). Additionally, social anxiety is considered to be one major cause of socially withdrawn and avoidance behavior, which are indispensable for leading to miss opportunities for normal socialization experiences (Book \& Randall, 2002; La Greca \& Stone, 1993), and this is one of the important reasons that why lonely feelings develop. There is a growing body of literature suggests a robust correlation between social anxiety and loneliness (Caplan, 2006; Johnson, LaVoie, Spenceri, \& MAHONEY-WERNLI, 2001; Neto \& Barros, 2000; Wilbert \& Rupert, 1986). Moreover, it is confirmed that individuals with high social anxiety experience higher loneliness than those with low social anxiety (Anderson \& Harvey, 1988; Moore \& Schultz Jr, 1983).

A theoretical model illustrating the hypothesized causal relationships between self-esteem, social anxiety and loneliness is presented in Figure 1. We hypothesized that: self-esteem predicts both social anxiety (path $a$ ) and loneliness (path $c^{\prime}$ ) negatively, and social anxiety positively predicts loneliness (path $b$ ). The aim of the current study is to investigate the mediated role of social anxiety for the relationship between self-esteem and loneliness. Moreover, several effect size measures are used to determine the effect size of the indirect effect. Findings from this study intend to provide promising support for uncovering critical components that address how to reduce loneliness.

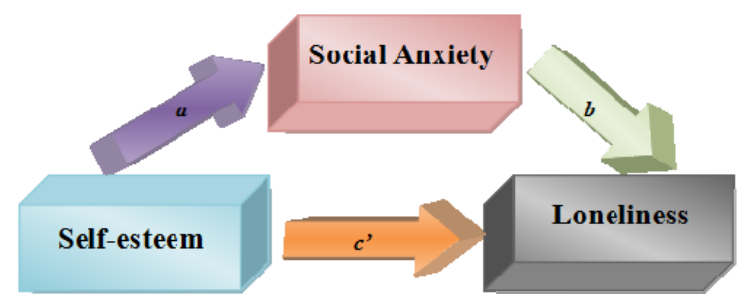

Figure 1. The theoretical model

\section{Method}

\subsection{Data Analysis Strategies}

The data collected in the current study were analyzed using the SPSS 19.0 and Mplus 7.0 statistical programs. First, data were collected by using the Rosenberg Self Esteem Scale, Interaction Anxiousness Scale and UCLA Loneliness Scale-Version 3. The internal consistencies of all the measuring scales were checked by the Cronbach's alpha coefficients and composite reliability. The normality of the scores of the self-esteem, the social anxiety and the loneliness were assessed with the Quantile-Quantile plot test and skewness and kurtosis measurements. Pearson correlation was used to assess the correlational relationships between the self-esteem, the social anxiety and the loneliness. The causal steps approach suggested by Baron and Kenny was employed to test the hypothesis that social anxiety mediates the relationship between self-esteem and loneliness. Furthermore, the significance of the indirect effect was assessed by both bootstrapping method and Markov chain Monte Carlo 
method. After the mediating effect had analyzed, the Kappa-squared and R-squared mediation effect size measures and 4 additional effect size measures, the partially standardized indirect effect, the completely standardized indirect effect, the proportion mediated and the ratio effect size measure were used to evaluate the effect size of the indirect effect.

\subsection{Participants \& Procedures}

Participants were drawn from a university located in a southern city of China. Three research assistants were recruited in the current study, and they were asked to distribute the informed consent forms and questionnaires to the participating students. In turn, participants were each given a small gift, and research assistants were each given $¥ 100$. Ninety-six percent of the students returned the signed consent forms. The final sample consisted of 285 students with 124 males and 161 females. Female students made up $56.5 \%$ of the sample. The age of the students averaged 20.01 $(S D=1.69)$ years.

\subsection{Measures}

\subsubsection{Rosenberg Self Esteem Scale}

The Rosenberg Self Esteem Scale, developed by Rosenberg, is the most widespread scale for measuring global feelings of self-worth or acceptance (Rosenberg, 1989). This scale consists of 10 items and respondents indicate their agreement with statements on a 4-point Likert scale $(1=$ strongly agree, $2=$ agree, $3=$ disagree, $4=$ strongly disagree). High scores, on the scale, indicate high self-esteem. The RSES has a reported Cronbach's alpha for internal consistency ranging from 0.82 to 0.88 , and a two-week test-retest reliability was reported as 0.72 (Warkentin, 2008).

\subsubsection{Interaction Anxiousness Scale}

The social anxiety was measured by the Interaction Anxiousness Scale (Leary, 1983). Interaction Anxiousness Scale is composed of 15 items, respondents are required to indicate their agreement with statements on a 5-point Likert-type scale $(1=$ not at all characteristic, $2=$ slightly characteristic, $3=$ moderately characteristic, $4=$ very characteristic, 5 = extremely characteristic.). The Interaction Anxiousness Scale measures the global feeling of social anxiety, higher scores mean higher social anxiety. The Interaction Anxiousness Scale has demonstrated sound psychometric qualities. The internal consistency reliability indices ranging from 0.87 to 0.89 have been reported (Leary \& Kowalski, 1993). Furthermore, test-retest reliability index is reported as 0.83 (Leary, 1983).

\subsubsection{UCLA Loneliness Scale-Version 3}

The UCLA Loneliness Scale-Version 3 was used to measure the participants' loneliness (Russell, 1996). UCLA Loneliness Scale is composed of 20 items, and participants rate their agreement from 1 (not at all true of me) to 4 (very true of me) on a 4-point Likert-type scale. It has been proved that the UCLA Loneliness Scale-Version 3 is a reliable scale in measuring one's loneliness, whose Chronbach's alpha coefficient is reported as ranging from 0.89 to 0.94 across samples and test-retest reliability is 0.73 (Russell, 1996).

\section{Results}

\subsection{Cronbach's Alpha \& Composite Reliability Analysis}

Cronbach's alpha tests were conducted to measure the level of consistency among the items in each scale. Results showed that the Cronbach's alpha coefficients for Rosenberg Self Esteem Scale, Interaction Anxiousness Scale and UCLA Loneliness Scale were 0.754, 0.831 and 0.873 respectively, indicating that the measuring scales were reliable (George, 2003; Yu, 2001).

Though the Cronbach's alpha coefficients of the three variables ranging from 0.754 to 0.873 , for rigorous, composite reliability (CR) of each scale was also calculated for assessing the internal consistency of the data collected (Raykov, 1997). Fan and Thomson (Fan \& Thompson, 2001) suggested that the confidence interval should also be reported along with a point estimate of CR. Bootstrapping method is a commonly used technique for constructing confidence interval for CR.

According to Raykov (Raykov \& Marcoulides, 2010), to obtain a bootstrapping confidence interval for composite reliability, thousands of samples should be drawn from the original data and then estimating composite reliability in each of the samples, and a bootstrapping confidence interval of reliability will be constructed. Because the bias-corrected bootstrapping has several advantages (Raykov \& Marcoulides, 2010), in the current study, 5000 samples were drawn from our original samples, and the bias-corrected $95 \%$ confidence intervals for the composite reliabilities of the Rosenberg Self Esteem Scale, Interaction Anxiousness Scale and UCLA Loneliness Scale-Version 3 were constructed respectively. 
Results were presented in Table 1 . The point estimate of each composite reliability and the lower limit of every constructed $95 \%$ confidence interval all exceeded 0.7 , suggesting that the scores of self-esteem, social anxiety and loneliness were internally consistent (Johari, Yahya, \& Omar, 2011).

Table 1 . Composite reliability and $95 \%$ CIs for all measures

\begin{tabular}{llll}
\hline & Composite Reliability & BC Bootstrap LLCI & BC Bootstrap ULCI \\
\hline Rosenberg Self Esteem Scale & 0.773 & 0.726 & 0.820 \\
Interaction Anxiousness Scale & 0.835 & 0.798 & 0.872 \\
UCLA Loneliness Scale & 0.875 & 0.848 & 0.902 \\
\hline
\end{tabular}

\subsection{Preliminary Descriptive Analysis}

Table 2 showed us that the self-esteem scores ranged from 20 to 39, and as presented in Figure 2, most of the points felt nicely on a straight line, indicating that the self-esteem scores were normally distributed. The social anxiety scores ranged from 24 to 76, and Figure 3 showed a normal distribution trend of the social anxiety scores with skewness $=0.271$ and kurtosis $=0.875$. The loneliness scores ranged from 20 to 80 , and as presented in Figure 3, a normal distribution trend of the loneliness scores was found with skewness $=0.130$ and kurtosis $=$ 0.441

Table 2. Descriptive statistics for all measures

\begin{tabular}{llllllll}
\hline & $\mathrm{N}$ & Min & Max & M & SD & Skewness & Kurtosis \\
\hline Self-esteem & 285 & 20 & 39 & 28.48 & 3.37 & 0.198 & 0.224 \\
Social anxiety & 285 & 24 & 76 & 49.53 & 7.87 & 0.271 & 0.857 \\
Loneliness & 285 & 20 & 80 & 43.08 & 8.55 & 0.130 & 0.441 \\
\hline
\end{tabular}

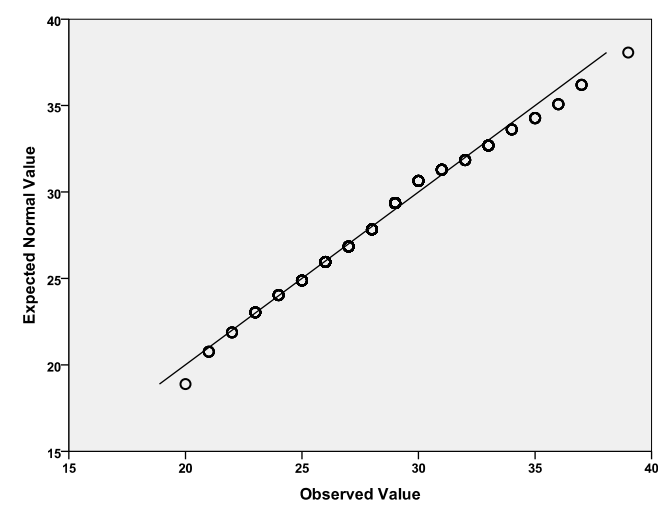

Figure 2. Normal Q-Q plot of the Self-esteem Score 


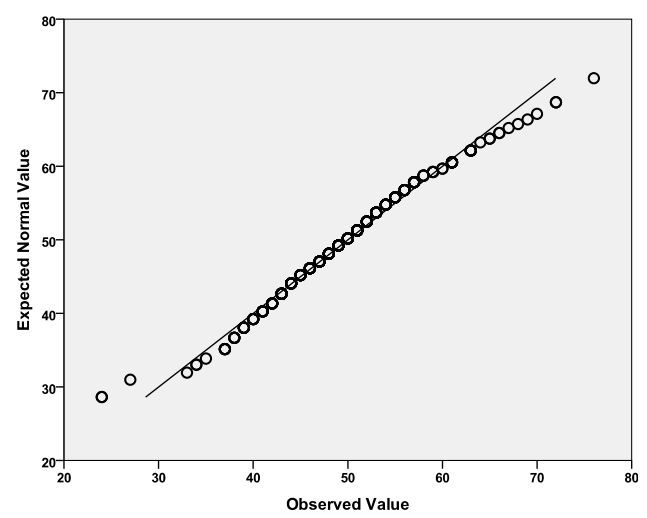

Figure 3. Normal Q-Q plot of the social anxiety score

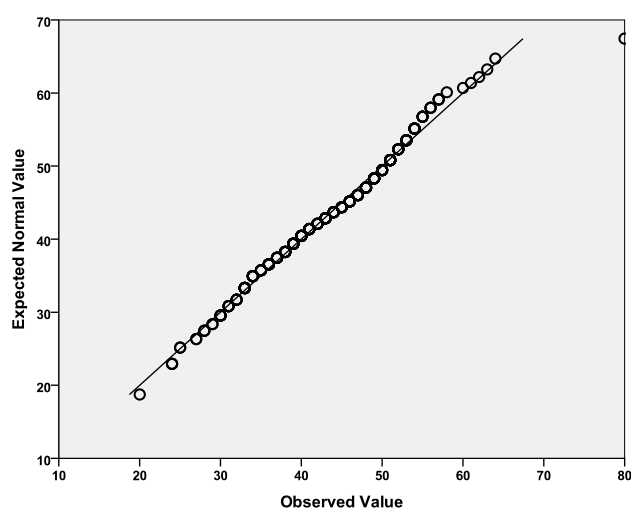

Figure 4. Normal Q-Q plot of the loneliness score

\subsection{Simple Correlation Analysis}

The data collected in the current study were first plotted in a 3D-scatter plot, assigning "self-esteem" to the $X$ axis, and "social anxiety" to the $Z$ axis and "loneliness" to the $Y$ axis. This 3D-scatter plot was used to determine the relationships between self-esteem, social anxiety and loneliness preliminarily. Figure 5 showed a 3D-scatter plot of the relationships between self-esteem, social anxiety and loneliness, which indicated that there were linear relationships between pairs of these three variables.

In order to find out the strength of the linear relationships, Pearson correlation analysis was conducted, and 5000 bootstrap samples were drawn from the original data to construct the bias-corrected $95 \%$ confidence intervals for all the correlation coefficients (Lunneborg, 1985). Results were presented in Table 3, self-esteem showed negative, significant relationships with social anxiety $(r=-0.422, p=0.000,95 \% \mathrm{CI}=-0.524,-0.306)$ and loneliness $(r=-0.444, p=0.000,95 \% \mathrm{CI}=-0.569,-0.271)$, however, social anxiety showed positive, significant relation with loneliness $(r=0.412, p=0.000,95 \% \mathrm{CI}=0.307,0.512)$. 


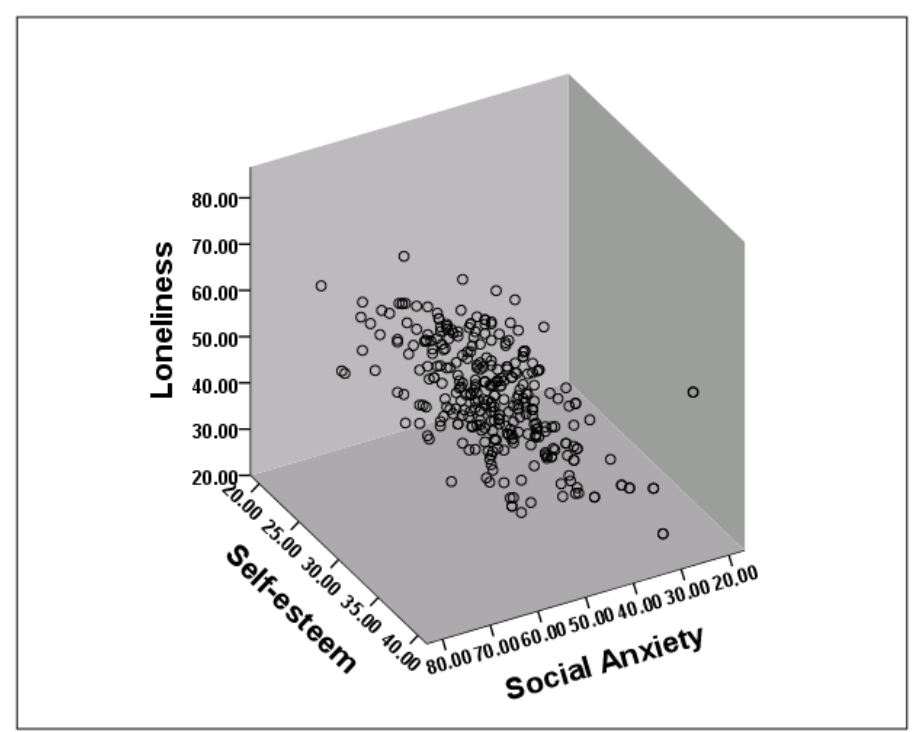

Figure 5. The 3D-scatter plot

Table 3. Correlation of self-esteem, social anxiety and loneliness variables

\begin{tabular}{llll}
\hline & Self-esteem $(X)$ & Social anxiety $(M)$ & Loneliness $(Y)$ \\
\hline Self-esteem $(X)$ & 1 & $-0.422^{* *}$ & $-0.444^{* * *}$ \\
Social anxiety $(M)$ & $-0.422^{* *}$ & 1 & $0.412^{* * *}$ \\
Loneliness $(Y)$ & $-0.444^{* * *}$ & $0.412^{* * *}$ & 1 \\
\hline
\end{tabular}

Note. ${ }^{* *} P<0.01$

\subsection{Mediation Analysis}

Baron and Kenny (Baron \& Kenny, 1986) proposed that several conditions should be met when testing a mediating effect: (1) the independent variable self-esteem predicts the dependent variable loneliness, the total effect of self-esteem on loneliness (path coefficient $c$ ) should be significant; (2) the independent variable self-esteem predicts the mediator social anxiety, the effect of self-esteem on social anxiety (path coefficient $a$ ) be significant; (3) The effect of social anxiety on loneliness controlled for self-esteem (path coefficient $b$ ) must be significant and (4) The direct effect of self-esteem on loneliness adjusted for social anxiety (path coefficient $c^{\prime}$ ) must be non-significant. Models in which all four steps are satisfied are called fully mediated models. A model in which Step 4 is relaxed so that the requirement is only $\left|c^{\prime}\right|<|c|$, rather than that $\left|c^{\prime}\right|$ be non-significant, is called a partially mediated model (Fritz \& MacKinnon, 2007; MacKinnon, Lockwood, Hoffman, West, \& Sheets, 2002). Moreover, Preacher and Hayes suggested not reporting standardized coefficients for mediation analysis (Preacher \& Hayes, 2008).

According to the causal steps approach suggested by Baron and Kenny, the first regression was conducted to investigate the total effect of self-esteem on loneliness. Results showed that self-esteem had a unique effect on loneliness, the total effect of self-esteem on loneliness was significant (Unstandardized Regression Coefficient $\left.\left(B_{c}\right)=-1.126, S E=0.135, t=-8.356, P=0.000,95 \% \mathrm{CI}=-1.390,-0.862\right)$.

The second regression was conducted to test whether self-esteem predicts the mediator social anxiety. Results showed that self-esteem was negatively associated with social anxiety (Unstandardized Regression Coefficient $\left.\left(B_{a}\right)=-0.986, S E=0.126, t=-7.848, P=0.000,95 \% \mathrm{CI}=-1.232,-0.739\right)$.

The effect of social anxiety on loneliness controlled for self-esteem was significant. Results showed that loneliness regressed significantly on social anxiety (Unstandardized Regression Coefficient $\left(B_{b}\right)=0.297, S E=$ $0.061, t=4.869, P=0.000,95 \% \mathrm{CI}=0.178,0.417)$, and the direct effect of self-esteem on loneliness adjusted for social anxiety was also found to be significant (Unstandardized Regression Coefficient $\left(B_{c^{\prime}}\right)=-0.833, S E=0.143$, $t=-5.833, P=0.000,95 \% \mathrm{CI}=-1.113,-0.553)$. Because the direct effect remained significant and smaller than 
the total effect $(|-0.833|<|-1.126|)$, which indicated that the mediation model in the current study was a partially mediated model.

Although the causal steps approach is commonly used for testing a mediating effect, simulation study showed that the causal steps approach has the lowest statistical power and may underestimate Type I error under certain conditions (MacKinnon et al., 2002; MacKinnon, Lockwood, \& Williams, 2004) (MacKinnon, Fairchild, \& Fritz, 2007). Moreover, Fritz and MacKinnon found that the causal steps approach requires a large number of subjects (e.g., 21,000) to reach 0.8 power to detect significant mediating effects (Fritz \& MacKinnon, 2007). According to Preacher and Hayes (Preacher \& Hayes, 2004, 2008) and Mackinnon (MacKinnon et al., 2002), only when the sample size is large, the results generated by causal steps approach can be accepted.

Recent years, researchers such as Mackinnon, Preacher and Hayes suggested and advocated using bootstrapping method as a robust means for assessing significance of the indirect effect, and this method has been proved to perform best in terms of both power and controlling Type I error rates (Hayes, 2009; MacKinnon et al., 2002; Preacher \& Hayes, 2004; Preacher, Rucker, \& Hayes, 2007).

Bootstrapping is a nonparametric resampling technique which does not impose the assumption of normality of the sampling distribution (Preacher \& Hayes, 2008; Preacher \& Selig, 2012). The bootstrapping method draws $n$ samples, say 5000 samples in this study, with replacement from the original sample, such as 285 samples in the current study, and then re-estimate mediation model and calculate the indirect effect $(a b)$ within each bootstrapping sample, and the percentile confidence intervals, the bias-corrected confidence intervals of the indirect effect will be constructed. If zero does not include in the confidence intervals, a conclusion that the indirect effect is significantly different from zero at $P<0.05$ can be drawn.

However, several researches found under certain conditions that bootstrapping will elevate type I error (Fritz \& MacKinnon, 2007; MacKinnon et al., 2004). Furthermore, the interpretation of confidence interval is based on repeated sampling, which involves repeating the same experiment under the exact same conditions many times. However, it is not the case in practice which is almost impossible to be conducted in the actual social science studies (Yuan \& MacKinnon, 2009).

Yuan and Mackinnon suggested using MCMC method to construct Bayesian credible intervals for assessing the significance of indirect effects for the reason that the interpretations of Bayesian credible intervals are more natural than the confidence intervals (Yuan \& MacKinnon, 2009). Under the Bayesian approach, one advantage of the MCMC method is that inferences can allow the usage of "Priors" of model parameters to incorporate known information about the model from sources other than the data being analyzed. The efficiency of the sampling process will be influenced if prior information is utilized. One can also specify the non-informative MCMC method to allow the model to be estimated directly using the original data (Yuan \& MacKinnon, 2009).

Simulation study showed that when the indirect effect was zero $(a=b=0)$, the coverage rate of Bayesian $95 \%$ credible intervals were larger than the nominal value of $95 \%$. Moreover, when sample size was larger than 200 , both the Bayesian non-informative prior and informative prior $95 \%$ credible intervals provided coverage rates of $100 \%$, which means that the estimated Type I error rate was 0 . Bradley proposed a criterion that Type I error rate should range between 0.025 and 0.075 (Bradley, 1978) and thus we assume that under certain conditions, the MCMC method may underestimate Type I error.

As noted previously that the bootstrapping method may overestimate Type I error and the MCMC method may underestimate Type I error, in order to control the type I error and elevate the statistical power, a cross validation strategy was employed (Cheung \& Lau, 2008). In the first step, 5000 samples were drawn from the original samples to construct both the percentile and bias-corrected $95 \%$ confidence intervals for the indirect effect. In the second step, the non-informative MCMC method was used for constructing the $95 \%$ credible interval of the indirect effect. Moreover, the $95 \%$ credible interval of the indirect effect was also constructed by the informative MCMC method. The prior information we used was obtained from the hierarchical regression analysis that previously conducted, and the regression parameters in the constructed model were limited using these regression coefficients. In the Bayesian approach, two Markov chains were used, and 10,000 posterior samples of the model parameters were drawn after 1,000 burn-in iterations to make inferences.

The mean estimate of the indirect effect $a b$ was $-0.293(S E=0.071, t=-4.137, P=0.000,95 \% \mathrm{CI}=-0.432$, -0.154 ), and the results of the $95 \%$ confidence intervals of the indirect effect were presented in Table 4 . The percentile confidence interval and bias-corrected confidence interval of the indirect effect were $[-0.456,-0.140]$ and $[-0.476,-0.156]$ respectively, which suggested that the indirect effect was significant. Table 5 presented the $95 \%$ credible intervals of the indirect effect. The results showed us that the $95 \%$ credible intervals of $a b$ constructed by the non-informative and informative MCMC methods were [-0.442, -0.164$]$ and $[-0.395,-0.200]$ 
respectively. The indirect effect was considered statistically significant as zero does not contain in both the $95 \%$ confidence intervals and the $95 \%$ credible intervals.

Table $4.95 \%$ confidence intervals constructed by percentile and bias-corrected bootstrapping methods

\begin{tabular}{lllllll}
\hline \multirow{2}{*}{ Effect } & \multicolumn{2}{l}{ Percentile Bootstrapping } & \multicolumn{3}{c}{ Bias-corrected Bootstrapping } \\
\cline { 2 - 6 } & Estimate & LLCI & ULCI & Estimate & LLCI & ULCI \\
\hline$a b$ & -0.293 & -0.456 & -0.140 & -0.293 & -0.476 & -0.156 \\
Total effect & -1.126 & -1.457 & -0.830 & -1.126 & -1.435 & -0.719 \\
Direct effect & -0.833 & -1.220 & -0.404 & -0.833 & -1.189 & -0.359 \\
\hline
\end{tabular}

Table $5.95 \%$ credible intervals constructed by non-informative and informative MCMC methods

\begin{tabular}{lllllllll}
\hline \multirow{2}{*}{ Effect } & \multicolumn{3}{c}{ Non-informative MCMC } & \multicolumn{4}{c}{ Informative MCMC } \\
\cline { 2 - 9 } & Estimate & Posterior SD & LLCI & ULCI & Estimate & Posterior SD & LLCI & ULCI \\
\hline$a b$ & -0.291 & 0.071 & -0.442 & -0.164 & -0.293 & 0.050 & -0.395 & -0.200 \\
Total effect & -1.128 & 0.136 & -1.396 & -0.856 & -1.127 & 0.133 & -1.387 & -0.865 \\
Direct effect & -0.832 & 0.144 & -1.114 & -0.550 & -0.832 & 0.137 & -1.102 & -0.565 \\
\hline
\end{tabular}

\subsection{Mediation Effect-Size Measure}

The proportion mediated, which gives what proportion the total effect is mediated by the mediator $(a b / c)$, is the most commonly-used effect-size measure for mediation (Alwin \& Hauser, 1975; MacKinnon \& Dwyer, 1993). However, the proportion mediated has been criticizing for its instability and bias in small samples (Freedman, 2001; MacKinnon, Warsi, \& Dwyer, 1995). Furthermore, if the total effect is small, the proportion mediated can be large, and only with a sample size that greater than 500 can proportion mediated perform well. Another alternative is the ratio effect size measure $\left(a b / c^{\prime}\right)$, which compares the mediated effect to the direct effect, but this measure is also limited by instability in small samples (MacKinnon et al., 1995).

Preacher suggested using $K^{2}$ (Kappa-squared) as a measure of effect size for the mediating effect (Preacher \& Kelley, 2011) while Fairchild and Mackinnon recommended the use of $R^{2}$ med (R-squared mediation effect size) for the measurement of mediating effect (Fairchild, MacKinnon, Taborga, \& Taylor, 2009). In order to evaluate the effect size of the mediating effect accurately, both the $K^{2}$ and $R^{2}{ }_{\text {med }}$ were calculated. Preacher suggested that it is no reason to report only one effect size and if circumstances permit, reporting multiple effect sizes to yield greater understanding of a given effect. Therefore, we also had the other four effect size measures, the partially standardized indirect effect, the completely standardized indirect effect, the proportion mediated and the ratio effect size measure, to be reported along with $K^{2}$ and $R_{\text {med }}^{2}$. Preacher also suggested reporting confidence interval for any point estimate effect size measure (Preacher \& Kelley, 2011). In light of this, 5000 bootstrap samples were drawn and $95 \%$ confidence intervals for all point estimate effect size measures were constructed.

Preacher suggested that Kappa-squared can be interpreted using Cohen's guidelines defining small (0.01); medium (0.09) and large (0.25) effect sizes (Preacher \& Kelley, 2011). In the current study, the point estimate of $K^{2}$ was $0.117(95 \% \mathrm{CI}=0.064,0.180)$. According to Cohen's guidelines, this point estimate of $K^{2}$ can be interpreted as a medium effect size and because the $95 \%$ confidence interval of $K^{2}$ excluded 0.01 and 0.25 but contained 0.09 , it was suggested that the mediating effect size of self-esteem on loneliness through social anxiety was medium. The point estimate of $R^{2}$ med was $0.108(95 \% \mathrm{CI}=0.056,0.179)$, indicating that the value of $R^{2}{ }_{\text {med }}$ was larger than $10 \%$ of the variance in loneliness was attributable to the indirect effect of self-esteem through social anxiety. The point estimate of $R_{\text {med }}^{2}$ was considered as a medium effect size. Additionally, the $95 \%$ confidence interval of $R^{2}$ med showed that at least $5.6 \%$ of the variance of loneliness were attributable to self-esteem mediated by social anxiety and up to $17.9 \%$ of the variance in loneliness is explained by the mediating effect. 
The partially standardized indirect effect was $-0.034(95 \% \mathrm{CI}=-0.054,-0.019)$, which implied that loneliness was expected to decrease by 0.034 standard deviations for every one-unit increase in self-esteem (on its 4-point scale) indirectly through social anxiety. The completely standardized indirect effect was $-0.115(95 \% \mathrm{CI}=-0.184$, -0.062 ), indicating that loneliness was expected to decrease by 0.115 standard deviations for every one standard deviation increase in self-esteem (on its 4-point scale) indirectly via social anxiety. The proportion that the total effect of self-esteem on loneliness was mediated by social anxiety and the ratio of the indirect effect to the direct effect of self-esteem on loneliness were $0.260(95 \% \mathrm{CI}=0.128,0.522)$ and $0.352(95 \% \mathrm{CI}=0.146,1.018)$ respectively. All the results presented above were in support of the conclusion that the effect size of the mediating effect of self-esteem on loneliness through social anxiety was medium.

\section{Discussion}

The current study examined the mediated role of social anxiety for the relationship between self-esteem and loneliness. In support of our study hypotheses, the current findings provided the evidence for a mediational model in which loneliness was associated with low self-esteem and high social anxiety, and that the relationship between self-esteem and loneliness was mediated by social anxiety. The findings from the meditational model were consistent with previous research, suggesting that low self-esteem was an antecedent cause of loneliness (Peplau et al., 1982) and a risk factor for social anxiety (De Jong et al., 2012), and that social anxiety in particular played a significant role in contributing to loneliness (Antony \& Swinson, 2008; Rokach, 2013). Kappa-squared (Preacher \& Kelley, 2011) and R-squared mediation effect size (Fairchild et al., 2009), the two new methods of expressing effect size for mediation effects were calculated to yield greater understanding of the indirect effect. Both the point estimates of $K^{2}$ and $R^{2}$ med and the confidence intervals of $K^{2}$ and $R_{\text {med }}^{2}$ suggested a medium effect size of the indirect effect, and this results were confirmed by the other 4 effect size measures.

The present study confirmed that self-esteem was negatively associated with loneliness, which was consistent with previous studies (Creemers et al., 2012; Kong \& You, 2013; McWhirter, 1997; McWhirter et al., 2002; Nurmi et al., 1997; Peplau et al., 1982). The self-esteem we measured in the current study was explicit self-esteem. According to the cognitive theory (Clak \& Beck, 1999), more internalizing symptoms are shown in people with low explicit self-esteem. Furthermore, the self-relevant information may process in a typical negative manner due to dysfunctional self-schemas existing in memory among individuals with low self-esteem. As a result, negative self-evaluations occurs which later lead to the development of loneliness (Creemers et al., 2012).

In this study, we confirmed that those with lower self-esteem have higher social anxiety, which was consistent with previous research (Guindon, 2009). It is defined that self-esteem is a person's evaluation of self. Individuals with low self-esteem are not only highly attentive to their image but also worry about how others view them (Heatherton, Wyland, \& Lopez, 2003). Furthermore, the individual's poor interpersonal relationships and lack of confidence in social situations as well as social interaction problems are accounted for by the low self-esteem (Juth, Smyth, \& Santuzzi, 2008) (Valencia, 2009), and this is thought to be one particular reason that why people develop social anxiety. People who develop social anxiety are suffering from intense fear of evaluation in social or performance situation (Jefferys, 1997) and feel uncomfortable doing something in front of strangers (Association \& DSM-IV., 1994).

Self-esteem is a value judgment based on self knowledge, which may act as a sociometer. Sociometer theory proposes that the self-esteem is a gauge (or sociometer) of the effectiveness in social relations and interactions (Leary \& Downs, 1995; Leary, Tambor, Terdal, \& Downs, 1995). Individual experiences a reduction in state of self-esteem if he or she behaves in a way which increases the chance he or she will be rejected. Accordingly, self-esteem may serve as a sociometer of social acceptance or rejection (Leary, 2005). When cues implying rejection are detected, the sociometer system alerts the individuals via negative affect and motivates behavior to restore standing with others, and therefore self-esteem may have evolved as a mechanism for monitoring the likelihood of social exclusion (Leary \& Kowalski, 1997).

It is common that the one's anxiety may occur as a response when one's sense of inclusion or becomingness is threatened (Leary \& Kowalski, 1997). The social anxiety may be the effective warning system of the sociometer which may play a significant role in alerting the individual when his or her social relationships are in danger and provide the impetus for taking immediate action (Leary \& Kowalski, 1997). As a result, it is no wonder that those with lower level of self-esteem have sociometer which indicates a high probability of rejection from others, and therefore they may worry about how they are being perceived by other people or their peers (Heatherton et al., 2003). Because of suspecting their own ability to intact with others in a desirable fashion (Geist \& Borecki, 
1982), individuals with low self-esteem feel more anxiety during social situations than those with high self-esteem.

One significant finding of the current study was that social anxiety mediates the relationship between self-esteem and loneliness and the effect size of the indirect effect was confirmed as medium, which illustrated that lower level of self-esteem leads to higher social anxiety and this may result in the development of loneliness. Loneliness appears as a result of being without some definitely essential relationship or set of relationships but not because of being alone (Weiss, 1973). Studies confirmed that if a person's network of social relations is significantly deficient both qualitatively and quantitatively, then as an unpleasant emotion, loneliness may be evoked (Perlman \& Peplau, 1981) (de Jong Gierveld, Van Tilburg, \& Dykstra, 2006).

It is no wonder that social anxiety occurs among individuals with low self-esteem as a result of perceiving high possibility of rejection from others. Social anxiety is considered to be one major cause of socially withdrawn and avoidance behavior, which are critical for leading to miss opportunities for normal socialization experiences (Book \& Randall, 2002; La Greca \& Stone, 1993). Since the man is by nature a social animal (Hursthouse, 1999; Vinciarelli, 2009; Vinciarelli et al., 2012), to form and maintain strong, stable interpersonal relationships with others are our basic desires and fundamental motivations (Baumeister \& Leary, 1995). When our intimate and social needs are not adequately met, loneliness, a complex set of feelings occurs (Baumeister \& Leary, 1995; Weiss, 1973).

There are several limitations in the current study that can be addressed in future research. First, the sample size in the current study is small. It is important to validate our study again based on a larger sample. Second, participants included a convenience sample of Chinese college students who were studying and living in a university that located in a southern city of China. Accordingly, the findings can not be extended to college students who study or live in other places of China. Furthermore, the participants in the current study were college students, and thus the findings can neither be extended to children nor older people. Third, data collected in the current study was not longitudinal data. It is unclear how much the long term effect self-esteem has on loneliness is mediated by social anxiety.

These limitations notwithstanding, the current findings provide empirical evidence regarding the relationships between self-esteem, social anxiety and loneliness. Moreover, the effect size of the mediating effect of self-esteem on loneliness through social anxiety is confirmed as medium. Furthermore, our findings provide promising support for uncovering critical components that address how to reduce loneliness.

\section{References}

Alwin, D. F., \& Hauser, R. M. (1975). The decomposition of effects in path analysis. American Sociological Review, 37-47. http://dx.doi.org/10.2307/2094445

Anderson, C. A., \& Harvey, R. J. (1988). Brief Report: Discriminating Between Problems in Living: An Examination of Measures of Depression, Loneliness, Shyness, and Social Anxiety. Journal of Social and Clinical Psychology, 6(3-4), 482-491. http://dx.doi.org/10.1521/jscp.1988.6.3-4.482

Antony, M., \& Swinson, R. (2008). The shyness and social anxiety workbook: Proven, step-by-step techniques for overcoming your fear. New Harbinger Publications.

Asher, S. R., \& Paquette, J. A. (2003). Loneliness and peer relations in childhood. Current Directions in Psychological Science, 12(3), 75-78. http://dx.doi.org/10.1111/1467-8721.01233

Association, A. P., \& DSM-IV., A. P. A. T. F. O. (1994). Diagnostic and statistical manual of mental disorders: $D S M-I V$. Amer Psychiatric Pub Inc.

Baron, R. M., \& Kenny, D. A. (1986). The moderator-mediator variable distinction in social psychological research: Conceptual, strategic, and statistical considerations. Journal of personality and social psychology, 51(6), 1173. http://dx.doi.org/10.1037/0022-3514.51.6.1173

Baumeister, R. F., \& Leary, M. R. (1995). The need to belong: Desire for interpersonal attachments as a $\begin{array}{lllll}\text { fundamental human motivation. Psychological bulletin, } & 117(3), & \end{array}$ http://dx.doi.org/10.1037/0033-2909.117.3.497

Book, S. W., \& Randall, C. L. (2002). Social anxiety disorder and alcohol use. Alcohol Research and Health, 26(2), 130-135.

Bradley, J. V. (1978). Robustness? British Journal of Mathematical and Statistical Psychology, 31(2), 144-152. http://dx.doi.org/10.1111/j.2044-8317.1978.tb00581.x 
Brage, D., Meredith, W., \& Woodward, J. (1993). Correlates of loneliness among midwestern adolescents. Adolescence.

Cacioppo, J. T., \& Patrick, W. (2008). Loneliness: Human nature and the need for social connection. WW Norton \& Company.

Caplan, S. E. (2006). Relations among loneliness, social anxiety, and problematic Internet use. Cyber Psychology \& Behavior, 10(2), 234-242. http://dx.doi.org/10.1089/cpb.2006.9963

Cheung, G. W., \& Lau, R. S. (2008). Testing mediation and suppression effects of latent variables bootstrapping with structural equation models. Organizational Research Methods, 11(2), 296-325. http://dx.doi.org/10.1177/1094428107300343

Chrystan, J. A. (2007). Social Rank and Social Anxiety. ProQuest.

Clak, D. A., \& Beck, A. T. (1999). Scientific foundations of cognitive theory and therapy of depression. Wiley. com.

Clark, D. A., \& Beck, A. T. (2011). Cognitive therapy of anxiety disorders: Science and practice. Guilford Publication.

Coplan, R. J., Closson, L. M., \& Arbeau, K. A. (2007). Gender differences in the behavioral associates of loneliness and social dissatisfaction in kindergarten. Journal of Child Psychology and Psychiatry, 48(10), 988-995. http://dx.doi.org/10.1111/j.1469-7610.2007.01804.x

Cox, B. J., Fleet, C., \& Stein, M. B. (2004). Self-criticism and social phobia in the US national comorbidity survey. Journal of Affective Disorders, 82(2), 227-234. http://dx.doi.org/10.1016/j.jad.2003.12.012

Creemers, D. H., Scholte, R. H., Engels, R. C., Prinstein, M. J., \& Wiers, R. W. (2012). Implicit and explicit self-esteem as concurrent predictors of suicidal ideation, depressive symptoms, and loneliness. Journal of behavior therapy and experimental psychiatry, 43(1), 638-646. http://dx.doi.org/10.1016/j.jbtep.2011.09.006

Cutrona, C. E. (1982). Transition to college: Loneliness and the process of social adjustment. Loneliness: A sourcebook of current theory, research, and therapy, 291-309.

Davis, S. F., Hanson, H., Edson, R., \& Ziegler, C. (1992). The relationship between optimism-pessimism, loneliness, and level of self-esteem in college students. College Student Journal.

De Jong Gierveld, J., Van Tilburg, T., \& Dykstra, P. A. (2006). Loneliness and social isolation. Cambridge handbook of personal relationships, 485-500. http://dx.doi.org/10.1017/CBO9780511606632.027

De Jong, P., Sportel, B., De Hullu, E., \& Nauta, M. (2012). Co-occurrence of social anxiety and depression symptoms in adolescence: differential links with implicit and explicit self-esteem? Psychological medicine, 42(3), 475. http://dx.doi.org/10.1017/S0033291711001358

Fairchild, A. J., MacKinnon, D. P., Taborga, M. P., \& Taylor, A. B. (2009). R 2 effect-size measures for mediation analysis. Behavior research methods, 41(2), 486-498. http://dx.doi.org/10.3758/BRM.41.2.486

Fan, X., \& Thompson, B. (2001). Confidence Intervals for Effect Sizes Confidence Intervals about Score Reliability Coefficients, Please: An EPM Guidelines Editorial. Educational and Psychological Measurement, 61(4), 517-531. http://dx.doi.org/10.1177/00131640121971365

Fordham, K., \& Stevenson-Hinde, J. (1999). Shyness, friendship quality, and adjustment during middle childhood. Journal of Child Psychology and Psychiatry, 40(5), 757-768. http://dx.doi.org/10.1111/1469-7610.00491

Freedman, L. S. (2001). Confidence intervals and statistical power of the "Validation" ratio for surrogate or intermediate endpoints. Journal of Statistical Planning and Inference, 96(1), 143-153. http://dx.doi.org/10.1016/S0378-3758(00)00330-X

Fritz, M. S., \& MacKinnon, D. P. (2007). Required sample size to detect the mediated effect. Psychological science, 18(3), 233-239. http://dx.doi.org/10.1111/j.1467-9280.2007.01882.x

Geist, C. R., \& Borecki, S. (1982). Social avoidance and distress as a predictor of perceived locus of control and level of self-esteem. Journal of clinical psychology, 38(3), 611-613. http://dx.doi.org/10.1002/1097-4679(198207)38:3<611::AID-JCLP2270380325>3.0.CO;2-H

George, D. (2003). SPSS for Windows Step by Step: A Simple Study Guide and Reference, 17.0 Update, 10/e. Pearson Education India. 
Guindon, M. H. (2009). Self-esteem across the lifespan: issues and interventions. CRC Press.

Hayes, A. F. (2009). Beyond Baron and Kenny: Statistical mediation analysis in the new millennium. Communication Monographs, 76(4), 408-420. http://dx.doi.org/10.1080/03637750903310360

Hayes, A. F., \& Preacher, K. J. (2010). Quantifying and testing indirect effects in simple mediation models when the constituent paths are nonlinear. Multivariate behavioral research, 45(4), 627-660. http://dx.doi.org/10.1080/00273171.2010.498290

Heatherton, T. F., Wyland, C. L., \& Lopez, S. (2003). Assessing self-esteem. Positive psychological assessment: A handbook of models and measures, 219-233.

Heinrich, L. M., \& Gullone, E. (2006). The clinical significance of loneliness: A literature review. Clinical psychology review, 26(6), 695-718. http://dx.doi.org/10.1016/j.cpr.2006.04.002

Hursthouse, R. (1999). Virtue ethics and human nature. Hume Studies, 25(1), 67-82.

Hymel, S., Rubin, K. H., Rowden, L., \& LeMare, L. (1990). Children's peer relationships: Longitudinal prediction of internalizing and externaliziing problems from middle to late childhood. Child Development, 61(6), 2004-2021. http://dx.doi.org/10.2307/1130854

Jefferys, D. (1997). Social phobia. The most common anxiety disorder. Australian family physician, 26(9), 1061, 1064-1067.

Johari, J., Yahya, K. K., \& Omar, A. (2011). The Construct Validity of Organizational Structure Scale: Evidence from Malaysia. World, 3(2), 131-152.

Johnson, H. D., LaVoie, J. C., Spenceri, M. C., \& MAHONEY-WERNLI, M. A. (2001). Peer conflict avoidance: Associations with loneliness, social anxiety, and social avoidance. Psychological Reports, 88(1), 227-235. http://dx.doi.org/10.2466/pr0.2001.88.1.227

Jones, W. H., Freemon, J., \& Goswick, R. A. (1981). The persistence of loneliness: Self and other determinants 1. Journal of Personality, 49(1), 27-48. http://dx.doi.org/10.1111/j.1467-6494.1981.tb00844.x

Juth, V., Smyth, J. M., \& Santuzzi, A. M. (2008). How do you feel? Self-esteem predicts affect, stress, social interaction, and symptom severity during daily life in patients with chronic illness. Journal of health psychology, 13(7), 884-894. http://dx.doi.org/10.1177/1359105308095062

Kirova-Petrova, A. (2000). Researching Young Children's Lived Experiences of Loneliness: Pedagogical Implications for Linguistically Diverse Students. Alberta Journal of Educational Research, 46(2), 99-116.

Knox, D., Vail-Smith, K., \& Zusman, M. (2007). The lonely college male. International Journal of Men's Health, 6(3), 273-279. http://dx.doi.org/10.3149/jmh.0603.273

Kong, F., \& You, X. (2013). Loneliness and self-esteem as mediators between social support and life satisfaction in late adolescence. Social Indicators Research, 110(1), 271-279. http://dx.doi.org/10.1007/s11205-011-9930-6

La Greca, A. M., \& Stone, W. L. (1993). Social anxiety scale for children-revised: Factor structure and concurrent validity. Journal of Clinical Child Psychology, 22(1), 17-27. http://dx.doi.org/10.1207/s15374424jccp2201_2

Larson, R. W. (1999). 12 The Uses of Loneliness in Adolescence. Loneliness in childhood and adolescence, 244. http://dx.doi.org/10.1017/CBO9780511551888.012

Leary, M. R. (1983). Social anxiousness: The construct and its measurement. Journal of personality assessment, 47(1), 66-75. http://dx.doi.org/10.1207/s15327752jpa4701_8

Leary, M. R. (2005). Sociometer theory and the pursuit of relational value: Getting to the root of self-esteem. European review of social psychology, 16(1), 75-111. http://dx.doi.org/10.1080/10463280540000007

Leary, M. R., \& Downs, D. L. (1995). Interpersonal functions of the self-esteem motive: The self-esteem system as a sociometer.

Leary, M. R., \& Kowalski, R. M. (1993). The interaction anxiousness scale: Construct and criterion-related

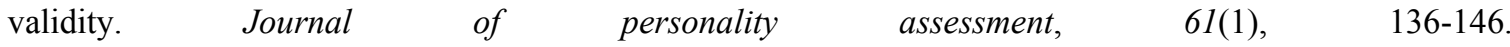
http://dx.doi.org/10.1207/s15327752jpa6101_10

Leary, M. R., \& Kowalski, R. M. (1997). Social anxiety. Guilford Publication.

Leary, M. R., Tambor, E. S., Terdal, S. K., \& Downs, D. L. (1995). Self-esteem as an interpersonal monitor: The 
sociometer hypothesis. Journal of personality and social psychology, 68(3), 518. http://dx.doi.org/10.1037/0022-3514.68.3.518

Lunneborg, C. E. (1985). Estimating the correlation coefficient: The bootstrap approach. Psychological bulletin, 98(1), 209. http://dx.doi.org/10.1037/0033-2909.98.1.209

MacKinnon, D. P., \& Dwyer, J. H. (1993). Estimating mediated effects in prevention studies. Evaluation review, 17(2), 144-158. http://dx.doi.org/10.1177/0193841X9301700202

MacKinnon, D. P., Fairchild, A. J., \& Fritz, M. S. (2007). Mediation analysis. Annual review of psychology, 58, 593. http://dx.doi.org/10.1146/annurev.psych.58.110405.085542

MacKinnon, D. P., Lockwood, C. M., Hoffman, J. M., West, S. G., \& Sheets, V. (2002). A comparison of methods to test mediation and other intervening variable effects. Psychological methods, 7(1), 83. http://dx.doi.org/10.1037/1082-989X.7.1.83

MacKinnon, D. P., Lockwood, C. M., \& Williams, J. (2004). Confidence limits for the indirect effect: Distribution of the product and resampling methods. Multivariate behavioral research, 39(1), 99-128. http://dx.doi.org/10.1207/s15327906mbr3901_4

MacKinnon, D. P., Warsi, G., \& Dwyer, J. H. (1995). A simulation study of mediated effect measures. Multivariate behavioral research, 30(1), 41-62. http://dx.doi.org/10.1207/s15327906mbr3001_3

Masi, C. M., Chen, H.-Y., Hawkley, L. C., \& Cacioppo, J. T. (2011). A meta-analysis of interventions to reduce loneliness. Personality and Social Psychology Review, 15(3), 219-266. http://dx.doi.org/10.1177/1088868310377394

McWhirter, B. T. (1997). Loneliness, learned resourcefulness, and self - esteem in college students. Journal of Counseling \& Development, 75(6), 460-469. http://dx.doi.org/10.1002/j.1556-6676.1997.tb02362.x

McWhirter, B. T., Besett-Alesch, T. M., Horibata, J., \& Gat, I. (2002). Loneliness in high risk adolescents: The role of coping, self-esteem, and empathy. Journal of Youth Studies, 5(1), 69-84. http://dx.doi.org/10.1080/13676260120111779

Mecca, A. M., Smelser, N. J., \& Vasconcellos, J. (1989). The social importance of self-esteem. University of California Pr.

Moore, D., \& Schultz Jr, N. R. (1983). Loneliness at adolescence: Correlates, attributions, and coping. Journal of Youth and Adolescence, 12(2), 95-100. http://dx.doi.org/10.1007/BF02088307

Neto, F., \& Barros, J. (2000). Psychosocial concomitants of loneliness among students of Cape Verde and Portugal. The Journal of psychology, 134(5), 503-514. http://dx.doi.org/10.1080/00223980009598232

Nurmi, J.-E., Toivonen, S., Salmela-Aro, K., \& Eronen, S. (1997). Social strategies and loneliness. The Journal of social psychology, 137(6), 764-777. http://dx.doi.org/10.1080/00224549709595497

O'Donovan, A., \& Hughes, B. (2007). Social support and loneliness in college students: effects on pulse pressure reactivity to acute stress. International journal of adolescent medicine and health, 19(4), 523-528. http://dx.doi.org/10.1515/IJAMH.2007.19.4.523

Obeid, N., Buchholz, A., Boerner, K. E., Henderson, K. A., \& Norris, M. (2013). Self-Esteem and Social Anxiety in an Adolescent Female Eating Disorder Population: Age and Diagnostic Effects. Eating disorders, 21(2), 140-153. http://dx.doi.org/10.1080/10640266.2013.761088

Olmstead, R., Guy, S., O'Mally, P., \& Bentler, P. M. (1991). Longitudinal assessment of the relationship between self-esteem, fatalism, loneliness, and substance use. Journal of Social Behavior \& Personality.

Oshagan, H., \& Allen, R. L. (1992). Three Loneliness Scales: An Assessment of Thier Measurement Properties. Journal of personality assessment, 59(2), 380-409. http://dx.doi.org/10.1207/s15327752jpa5902_13

Peplau, L. A., \& Cutrona, C. E. (1980). The revised UCLA Loneliness Scale: Concurrent and discriminant validity evidence. Journal of personality and social psychology, 39(3), 472-480. http://dx.doi.org/10.1037/0022-3514.39.3.472

Peplau, L. A., Perlman, D., Peplau, L. A., \& Perlman, D. (1982). Perspectives on loneliness. Loneliness: A sourcebook of current theory, research and therapy, 1-18.

Perlman, D., \& Peplau, L. A. (1981). Toward a social psychology of loneliness. Personal relationships, 3, 31-56.

Preacher, K. J., \& Hayes, A. F. (2004). SPSS and SAS procedures for estimating indirect effects in simple 
mediation models. Behavior Research Methods, Instruments, \& Computers, 36(4), 717-731. http://dx.doi.org/10.3758/BF03206553

Preacher, K. J., \& Hayes, A. F. (2008). Asymptotic and resampling strategies for assessing and comparing indirect effects in multiple mediator models. Behavior research methods, 40(3), 879-891. http://dx.doi.org/10.3758/BRM.40.3.879

Preacher, K. J., \& Kelley, K. (2011). Effect size measures for mediation models: Quantitative strategies for communicating indirect effects. Psychological methods, 16(2), 93. http://dx.doi.org/10.1037/a0022658

Preacher, K. J., Rucker, D. D., \& Hayes, A. F. (2007). Addressing moderated mediation hypotheses: Theory, methods, and prescriptions. Multivariate behavioral research, 42(1), 185-227. http://dx.doi.org/10.1080/00273170701341316

Preacher, K. J., \& Selig, J. P. (2012). Advantages of Monte Carlo confidence intervals for indirect effects. Communication Methods and Measures, 6(2), 77-98. http://dx.doi.org/10.1080/19312458.2012.679848

Raykov, T. (1997). Estimation of composite reliability for congeneric measures. Applied Psychological Measurement, 21(2), 173-184. http://dx.doi.org/10.1177/01466216970212006

Raykov, T., \& Marcoulides, G. A. (2010). Introduction to psychometric theory. Taylor \& Francis.

Rokach, A. (2011). From loneliness to belonging: a review. Psychology Journal, 8(0), 70-81.

Rokach, A. (2013). Loneliness Updated: Recent Research on Loneliness and how it Affects Our Lives. Routledge.

Rokach, A., \& Brock, H. (1997). Loneliness and the effects of life changes. The Journal of psychology, 131(3), 284-298. http://dx.doi.org/10.1080/00223989709603515

Rosenberg, M. (1989). Society and the adolescent self-image rev. Wesleyan University Press.

Russell, D. W. (1996). UCLA Loneliness Scale (Version 3): Reliability, validity, and factor structure. Journal of personality assessment, 66(1), 20-40. http://dx.doi.org/10.1207/s15327752jpa6601_2

Sarason, I. G., Sarason, B. R., \& Pierce, G. R. (1990). Social support: The search for theory. Journal of Social and Clinical Psychology, 9(1), 133-147. http://dx.doi.org/10.1521/jscp.1990.9.1.133

Schultz, N. R., \& Moore, D. (1988). Loneliness: Differences across three age levels. Journal of Social and Personal Relationships, 5(3), 275-284. http://dx.doi.org/10.1177/0265407588053001

Valencia, C. (2009). The Boost Your Self-esteem Workbook. Building Self Esteem.

Vanhalst, J. (2012). Loneliness in Adolescence: Developmental Course, Antecedents, and Consequences. http://dx.doi.org/10.1016/j.jadohealth.2011.07.006

Vinciarelli, A. (2009). Capturing order in social interactions [social sciences]. Signal Processing Magazine, IEEE, 26(5), 133-152. http://dx.doi.org/10.1109/MSP.2009.933382

Vinciarelli, A., Pantic, M., Heylen, D., Pelachaud, C., Poggi, I., D’Errico, F., \& Schröder, M. (2012). Bridging the gap between social animal and unsocial machine: A survey of social signal processing. Affective Computing, IEEE Transactions on, 3(1), 69-87. http://dx.doi.org/10.1109/T-AFFC.2011.27

Warkentin, J. B. (2008). Dating Violence and Sexual Assault Among College Men: Co-Occurence, Predictors, and Differentiating Factors. (Doctor of Philosophy), Ohio University.

Weiss, R. S. (1973). Loneliness: The experience of emotional and social isolation. Cambridge, Mass.: MIT Press.

Wilbert, J. R., \& Rupert, P. A. (1986). Dysfunctional attitudes, loneliness, and depression in college students. Cognitive Therapy and Research, 10(1), 71-77. http://dx.doi.org/10.1007/BF01173384

Yu, C. H. (2001). An introduction to computing and interpreting Cronbach Coefficient Alpha in SAS. Paper presented at the Proceedings of 26th SAS User Group International Conference.

Yuan, Y., \& MacKinnon, D. P. (2009). Bayesian mediation analysis. Psychological methods, 14(4), 301. http://dx.doi.org/10.1037/a0016972 


\section{Copyrights}

Copyright for this article is retained by the author(s), with first publication rights granted to the journal.

This is an open-access article distributed under the terms and conditions of the Creative Commons Attribution license (http://creativecommons.org/licenses/by/3.0/). 